\title{
A Case of Menstrual Psychosis, an Under Recognized Diagnosis
}

\author{
Woodward Kiel ${ }^{1}$, Wright Rhonda ${ }^{1,2}$ and Rathore Geetanjali ${ }^{1,2 *}$ \\ ${ }^{1}$ Department of Neurology, USA \\ ${ }^{2}$ Department of Pediatric Neurology, USA \\ *Corresponding author: Rathore Geetanjali, Department of Neurology and Pediatric Neurology, USA
}

\begin{abstract}
Menstrual psychosis is a rare disorder that causes psychotic symptoms in conjunction with a patient's menstrual cycle. We present a case of a 14-year-old girl who presents with such symptoms. Our patient presented with five days of somnolence, paranoia, and visual hallucinations soon after a diagnosis of streptococcal pharyngitis and being started on antimicrobial therapy. Her neurologic exam was significant for flat affect and psychomotor slowing. A thorough work-up consisting of brain MRI, EEG, LP, encephalopathy panel, along with metabolic, infectious, and inflammatory laboratory analysis was unremarkable, and she recovered without therapy about one week after symptom onset. Patient had recurrence and spontaneous resolution of her symptoms two more times over the next 10 months with repeat work-up negative; her family noted that the onset of her symptoms always seemed to coincide with the onset of her menses. She was started on combination oral contraceptive pills and has had no further recurrence of her symptoms since then.
\end{abstract}

\section{Background}

Menstrual psychosis is a rare diagnosis that has been declining in frequency over the past century. The presence of abnormal behavior suggestive of a psychological disorder associated with menstruation was first alluded to in the mid-18th century. Menstrual psychosis was first described in detail in the mid-19th century in France. There have been dozens of cases described up until the mid-20th century, with most detailed descriptions coming out of Germany, but only a smattering of case reports since then [1]. The decline in diagnoses may be related to skepticism of the diagnosis by the psychiatric community and declining awareness.

There are criteria that must be met before a diagnosis of menstrual psychosis can be applied, as described by Brockington $[1,2]$ :

a. Acute onset of symptoms in a previously asymptomatic patient

b. Brief duration with complete recovery

c. Psychotic symptoms: confusion, delusions, hallucinations, stupor, mutism, or mania d. Periodic with a portion of the patient's menstrual cycle

There does not seem to be an age that is affected more frequently than others, onset of symptoms can occur anytime from menarche to menopause. There are several other mood disorders that have been described to be related to the menstrual cycle, such as premenstrual syndrome, perimenopausal depression, and premenstrual dysphoric disorder. What defines menstrual psychosis apart from these other mood disorders is the presence of psychotic symptoms as mentioned above. There are five sub-types that have been described that are defined based on the portion of the menstrual cycle in which symptoms occur [2].

a. Premenstrual-Occurs during the second half of the menstrual cycle and may abruptly cease with the onset of menstrual bleeding.

b. Catamenial - Begins with onset of menstrual bleeding.

c. Mid-cycle - A less common variant that does not have a set relation to onset of menstrual flow.

d. Epochal - Lasts for the complete cycle, with onset/recovery linked to onset of menstrual flow. 
e. Premenstrual - The timing of symptom onset is less welldefined but is regularly linked with the menstrual cycle. Usually described in patients with irregular menstrual cycles.

\section{Case Presentation}

A 14-year-old white female with no significant past medical history presented with five days of somnolence, paranoia, and visual hallucinations soon after a diagnosis of streptococcal pharyngitis and being started on antimicrobial therapy. She commented that the office staff at her primary care physician were trying to harm her. She was admitted to the local pediatric hospital for further evaluation due to concern for underlying organic cause of her abnormal behavior. Mother notes that she has never exhibited this type of behavior before; she is typically very social, participates in cross-country running, and excels in school. There was no reported family history of mood disorder or psychosis. Her neurologic exam was significant for a flat affect and psychomotor slowing to the point of near-mutism but was otherwise unremarkable.

Laboratory work-up during this admission, including CBC, CMP, CRP, ESR, ammonia, TSH, free T4, urine drug screen, and urinalysis was unremarkable. Infectious work-up included EBV serology, which was negative, and mycoplasma serology, which showed a low positive titer of mycoplasma IgM. MRI brain with and without contrast showed a Chiari I malformation and mild third and lateral ventriculomegaly without evidence of acute obstruction. Routine EEG was normal. Lumbar puncture revealed CSF with normal protein and glucose, negative meningitis/encephalitis panel, negative autoimmune encephalopathy panel, WBC 2 , RBC $<1000$, and negative cytology. She completely recovered after about a week without therapy. No definite etiology was determined for her symptoms, she was discharged home to follow-up with her PCP.

Nine months later, the patient developed emotional lability and decreased concentration. She was brought to the local pediatric emergency department for evaluation. She had an unremarkable head CT and basic laboratory work-up, including urine drug screen. She was discharged home with instructions to return if her symptoms worsened. She returned to the ED three days later with continued complaints of "not feeling right" and visual hallucinations. During this admission, mother noted that her symptoms started on the same day as the onset of her menstrual cycle, as did her previous episode; the patient's menstrual cycle had been reportedly irregular, occurring only three times since her previous admission nine months ago. Repeat lumbar puncture, serum and CSF autoimmune encephalopathy antibody panels, and an anti-neuronal antibody panel were all negative. She had pelvic ultrasound to rule-out occult ovarian malignancy, which was negative. Once again, she spontaneously recovered six days after onset of symptoms without therapy.

One month later, the patient had another recurrence of her symptoms of somnolence, irritability that started soon after onset of menstrual cramping. As this was her third episode and she had complete spontaneous recovery after her previous encounters, her parents elected to watch at home. She was brought into the pediatric hospital two days later when patient started to display signs of possible visual hallucinations when she would wave her hands around in the air. Another thorough work-up, including pelvic MRI, was negative. Her symptoms, once again, resolved within seven days of onset. Her symptom onset coinciding with onset of menses on each occasion with repeated negative work-up of infectious or inflammatory cause raised suspicion of menstrual psychosis. She was started on combination norethindrone/ethinyl estradiol oral combination contraceptive pills and has had no further recurrence of symptoms since then.

\section{Discussion}

Despite the skepticism surrounding the diagnosis of menstrual psychosis, the role of the menstrual cycle and sex hormones in psychiatric disorders has been clearly demonstrated. One study showed that women who were admitted to a psychiatric hospital were more likely to be admitted during the premenstrual phase (3 days before and after onset of menses) than during other times of the menstrual cycle [3]. The underlying pathophysiology of menstrual psychosis is poorly understood but is thought to be related to the complex hormonal level fluctuations that occur during the menstrual cycle. There have been comparisons of menstrual psychosis to postpartum psychosis and hypotheses to link the two processes under the same pathophysiological process, as they are both rare disorders but several cases of patients suffering from both have been described [4].

A review of case reports describes several psychotic episodes in the context of not only naturally occurring times of estrogenwithdrawal, such as ovulation, onset of menses, the puerperium, and menopause, but also iatrogenic estrogen-withdrawal such as cessation of exogenous estrogens, administration of estrogenantagonists, and blockade of endogenous secretion of estrogen [5]. Attention has also been given to the role of progesterone, its metabolites, and other neuro steroids in the pathophysiology of psychosis. One study found an association between low levels of progesterone and first onset episode of psychosis, and that there may be an inverse correlation between the level of progesterone and the severity of the symptoms [6]. Progesterone and some of its metabolites, such as allopregnanolone and allotetra hydrodeoxy corticosterone (THDOC), have been noted to have anti-psychotic effects by enhancing modulation of GABAA receptors [7].

A similar link between changing hormonal levels during the menstrual cycle and neurological disease has been described before in patients with catamenial epilepsy; there have been studies that show anti-convulsant effects of the progesterone metabolites allopregnanolone and THDOC, the sudden withdrawal of progesterone that precedes menstrual bleeding leads to more 
frequent and severe seizures in these patients [8]. The effects of estrogens are less clear and have been shown to be both pro- and anti-convulsant [9]. While the use of typical and atypical neuroleptics has shown some usefulness for patients with menstrual psychosis, the established therapy for menstrual psychosis is regulation of hormonal levels during the menstrual cycle with oral contraceptive pills [10]. The use of exogenous estrogens and estrogen-modulation medications have been playing a larger role in other psychotic disorders, as well. Estrogen is thought to have a protective role in schizophrenia, as there is a well-established difference in age-ofonset, severity in symptoms, and response to treatment between males and females, which is commonly referred to as the "estrogen hypothesis" [11]. There have been several trials that have shown benefit of the use of estrogen and estrogen receptor-modulators, such as tamoxifen, in the long-term outcomes of patients with schizophrenia and treatment-resistant schizophrenia $[12,13]$.

\section{Conclusion}

Despite the rarity and skepticism surrounding the diagnosis of menstrual psychosis, there is growing evidence of a correlation between levels of estrogens, progesterone, and their metabolites on psychiatric syndromes. As research continues, menstrual psychosis and schizophrenia may be thought of as two entities in the same spectrum of disease. Until that time comes, the diagnosis of menstrual psychosis and treatment with menstrual cycle regulation should be kept in mind when evaluating women with treatmentresistant psychotic symptoms.

\section{References}

1. Brockington I (2005) Menstrual psychosis. World Psychiatry 4(1): 9-17.
2. Brockington I (2011) Menstrual psychosis-a bipolar disorder with a link to the hypothalamus. Current Psychiatry Reports. 13(3): 193-197.

3. Bergemann N, Parzer P, Nagl I, Salbach B, Runnebaum B, et al. (2002) Acute psychiatric admission and menstrual cycle phase in women with schizophrenia. Archives of Women's Mental Health 5(3): 119-126.

4. Deuchar N, Brockington I (1998) Puerperal and menstrual psychoses: The proposal of a unitary etiological hypothesis. Journal of Psychosomatic Obstetrics \& Gynecology 19(2): 104-110.

5. Mahé V, Dumaine A (2001) Oestrogen withdrawal associated psychoses. Acta Psychiatrica Scandinavica 104(5): 323-331.

6. Belvederi Murri M, anelli F, Pagotto U, Bonora E, Triolo F, et al. (2016) Neuroactive steroids in first-episode psychosis: A role for progesterone? Schizophrenia Research and Treatment.

7. Rupprecht R (2003) Neuroactive steroids: mechanisms of action and neuro psycho pharmacological properties. Psycho neuroendocrinology 28(2): 139-168.

8. Herzog A (2015) Catamenial epilepsy: Update on prevalence, pathophysiology, and treatment from the findings of the NIH Progesterone Treatment Trial. Seizure 28: 18-25.

9. Reddy D (2009) The role of neuro steroids in the pathophysiology and treatment of catamenial epilepsy. Epilepsy Research 85(1): 1-30.

10. Felthous A, Robinson D, Conroy R (1980) Prevention of recurrent menstrual psychosis by an oral contraceptive. American Journal of Psychiatry 137(2): 245-246.

11. Seeman M (1996) The role of estrogen in schizophrenia. Journal of Psychiatry and Neuroscience 21(2): 123-127.

12. Gogos A, Alyssa M Sbisa, Jeehae Sun, Andrew Gibbons, Madhara Udawela, et al. (2015) A role for estrogen in schizophrenia: clinical and preclinical findings. International Journal of Endocrinology.

13. Kulkarni J, Gavrilidis E, Wang W, Worsley R, Fitzgerald PB, et al. (2015) Estradiol for treatment-resistant schizophrenia: a large-scale randomized-controlled trial in women of child-bearing age. Molecular Psychiatry 20(6): 695-702.
(C) 9 This work is licensed under Creative

To Submit Your Article Click Here: Submit Article

DOI: $10.32474 /$ JJNBD.2018.02.000133

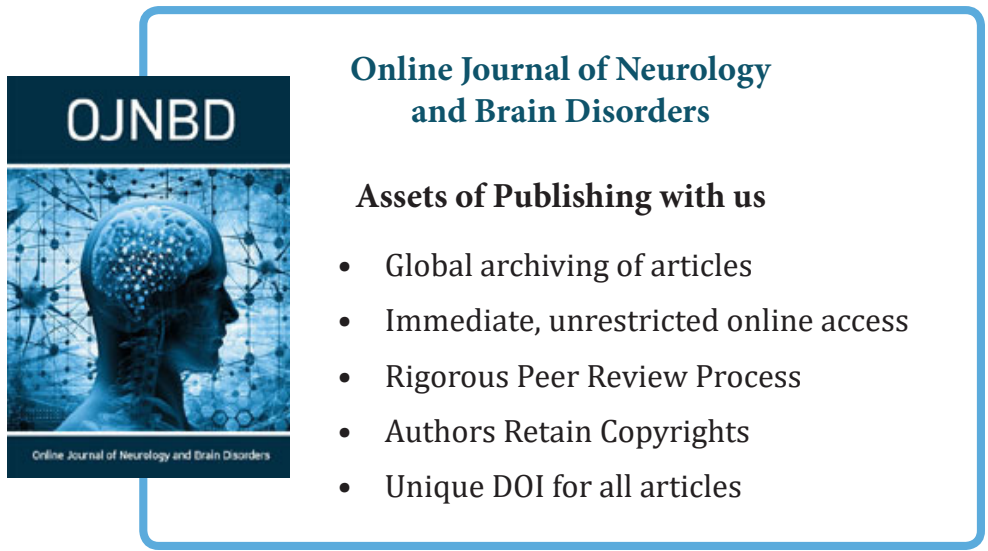

